\title{
Blue Luminescence and Extended Red Emission: Possible Connections to the Diffuse Interstellar Bands
}

\author{
A. N. Witt \\ Ritter Astrophysical Research Center, University of Toledo, Toledo, OH 43606, USA
}

\begin{abstract}
Blue luminescence (BL) and extended red emission (ERE) are observed as diffuse, optical-wavelength emissions in interstellar space, resulting from photoluminescence by ultraviolet(UV)-illuminated interstellar grains. Faintness and the challenge of separating the BL and ERE from the frequently much brighter dust-scattered continuum present major observational hurdles, which have permitted only slow progress in testing the numerous models that have been advanced to explain these two phenomena. Both the ERE, peaking near $680 \mathrm{~nm}$ (FWHM $\sim 60-120 \mathrm{~nm}$ ) and the BL, asymmetrically peaking at $\sim 378 \mathrm{~nm}($ FWHM $\sim 45 \mathrm{~nm})$, were first discovered in the Red Rectangle nebula. Subsequently, ERE and BL have been observed in other reflection nebulae, and in the case of the ERE, in carbon-rich planetary nebulae, H II regions, high-latitude cirrus clouds, the galactic diffuse ISM, and in external galaxies. BL exhibits a close spatial and intensity correlation with emission in the aromatic emission feature at 3.3 micron, most likely arising from small, neutral polycyclic aromatic hydrocarbon (PAH) molecules. The spectral characteristics of the BL also agree with those of fluorescence by PAH molecules with 13 to 19 carbon atoms. The BL phenomenon is thus most readily understood as the optical fluorescence of small, UV-excited aromatic molecules. The ERE, by contrast, though co-existent with mid-IR PAH emissions, does not correlate with emissions from either neutral or ionized PAHs. Instead, the spatial ERE morphology appears to be strictly governed by the density of far-UV $(\mathrm{E} \geqslant 10.5 \mathrm{eV})$ photons, which are required for the ERE excitation. The most restrictive observational constraint for the ERE process is its exceptionally high quantum efficiency. If the ERE results from photo-excitation of a nano-particle carrier by photons with $\mathrm{E} \geqslant$ $10.5 \mathrm{eV}$ in a single-step process, the quantum efficiency exceeds $100 \%$. Such a process, in which one to three low-energy optical photons may be emitted following a single far-UV excitation, is possible in highly isolated small clusters, e.g. small, dehydrogenated carbon clusters with about 20 to 28 carbon atoms. A possible connection between the ERE carriers and the carriers of DIBs may exist in that both are ubiquitous throughout the diffuse interstellar medium and both have an abundance of low-lying electronic levels with $\mathrm{E} \leqslant 2.3 \mathrm{eV}$ above the ground state.
\end{abstract}

Keywords. ISM: dust, ISM: lines and bands: ISM: individual: Red Rectangle

\section{Introduction}

Despite decades of observational, theoretical, and experimental research with focus on precise wavelengths, equivalent widths, polarization, and detailed profiles of diffuse interstellar bands (DIBs), a generally convincing identification of the carriers of this phenomenon has remained elusive. The search for further constraints on the nature of the DIB carriers has led over time to investigations of possible correlations with other poorly understood interstellar phenomena that are generally attributed to the smallest of interstellar grains, such as the far-ultraviolet extinction rise, the $2175 \AA$ interstellar extinction bump (Witt et al. 1983; Seab \& Snow 1984; Xiang et al. 2011), the mid-infrared aromatic emission bands (Salama \& Allamandola 1994; Mulas et al. 2003), and the dust-related anomalous microwave emission (Iglesias-Groth 2008). Such studies were motivated by the 
fact that the abundances of interstellar grains and molecules are tightly constrained by the cosmic abundances of the elements and the observed depletions from the gas phase (Snow \& Witt 1995, 1996), especially for the crucial element carbon. It is, therefore, both likely and necessary that different interstellar phenomena are manifestations of the same carrier class. It is with this motivation that I want to examine whether the likely carriers of the optical dust luminescence phenomena Blue Luminescence (BL) and Extended Red Emission (ERE) could possibly be related to DIBs.

\section{The Observation of ERE and BL}

Despite the fact that both phenomena exhibit their spectral signatures in the optical wavelength range, ERE and BL were discovered comparatively late (Cohen et al. 1975; Schmidt et al. 1980; Vijh et al. 2004). Both forms of diffuse dust emission were discovered first in the proto-planetary Red Rectangle (RR) nebula, where ERE, peaking near $680 \mathrm{~nm}(\mathrm{FWHM} \sim 60-120 \mathrm{~nm})$, and BL, asymmetrically peaking at $\sim 378 \mathrm{~nm}(\mathrm{FWHM}$ $\sim 45 \mathrm{~nm})$, are exceptionally bright and the usually dominant scattered light continuum is exceptionally weak, thanks to an unfavorable scattering geometry.

The RR is powered by HD 44179, a binary consisting of a mass-losing post-AGB giant star and a mass-accreting solar-type main-sequence companion, hidden from direct observation by an optically thick, circum-binary dusty torus, which presents a nearly edge-on view to Earth-based observers. The dust in the RR is forming locally in the carbon-rich bi-conical outflow, driven by a jet arising from the accretion disk surrounding the secondary star (Witt et al. 2009; Thomas et al. 2013).

In the RR, the ERE is readily extracted by dividing the nebular spectrum by that of the illuminating star seen through scattering in the center of the nebula (Witt \& Boroson 1990). The emission nature of the ERE is confirmed by a corresponding decrease in the linear polarization of the nebular light over the wavelength extent of the ERE band (Schmidt et al. 1980; Watkin et al. 1991) due to the dilution of the otherwise more strongly polarized scattered light. The BL was first detected by noting the partial fillingin of the hydrogen Balmer series absorption lines in the nebular spectrum when compared to the Balmer line profiles seen in the spectrum of HD 44179, the central star (Vijh et al. 2004). With observations of Balmer lines with upper states $\mathrm{n}=4$ to $\mathrm{n}=13$, a reddegraded profile with a peak at $378 \mathrm{~nm}$ emerges.

The spatial morphologies of the ERE and the BL in the RR are distinctly different (Vijh et al. 2005a, 2006). While the BL is brightest relative to the scattered light in the dark shadow regions cast outside the circum-binary torus in the RR, the ERE is predominantly confined to the narrow boundary region on the inner walls of the bi-cone of the outflow from the center. These are the regions that are most directly illuminated by the central source of the nebula (Schmidt \& Witt 1991). The ERE is mainly responsible for the X-shape morphology of the RR, which is its identifying feature.

Subsequent to its discovery in the RR, ERE was observed extensively in reflection nebulae (Witt et al. 1984; Witt \& Boroson 1990), planetary nebulae (Witt \& Furton 1990, 1992), in H II regions (Perrin \& Sivan 1992; Darbon et al. 1998, 2000), in the diffuse ISM at high galactic latitudes (Guhathakurta \& Tyson 1989; Gordon et al. 1998; Szomoru \& Guhathakurta 1998; Witt et al. 2008; Matsuoka et al. 2011), and in external galaxies (Perrin et al. 1995; Pierini et al. 2002).

Similarly, using the same technique that led to the detection of the BL in the RR, Vijh et al. (2005b) found BL in other reflection nebulae illuminated by early-type stars. 


\section{Carriers of the $\mathrm{BL}$}

The BL is most likely the result of fluorescence by small, neutral PAH molecules with three to four aromatic rings, in particular the molecules anthracene, phenanthrene, and pyrene. This suggestion (Vijh et al. 2005a) is supported by several observed characteristics of the BL in the RR. These characteristics are:

(a) The BL spectrum closely matches the laboratory fluorescence spectra of these three molecules;

(b) the spatial morphology of the BL is very similar to that of the aromatic $\mathrm{C}-\mathrm{H}$ stretch band at 3.3 micron (Kerr et al. 1999);

(c) the BL emitters distinctly prefer regions of the RR nebula that are shielded against direct illumination by the central source, and are missing where the UV field from the central source would lead to ionization of such molecules.

Theoretical models of the mid-infrared emission bands from neutral and ionized PAHs by Bakes et al. (2001) support this identification by showing that small neutral PAHs exhibit strong 3.3-micron bands, while these bands are not seen in the spectra of corresponding cations.

The identification of the BL spectrum with that of fluorescence by small, neutral PAHs does not, however, exclude the presence of larger PAHs and small, PAH-related molecules. Thomas \& Witt (2006) showed that the gas-phase fluorescence spectrum of phenanthridine, a three-ringed PAH with a single nitrogen substitution, also provides an excellent fit to the BL spectrum. Furthermore, the gas-phase fluorescence spectrum of a mixture of similar quantities of both small and large PAHs is strongly dominated by the fluorescence by three- and four-ringed PAHs, which appear to have significantly higher fluorescence efficiencies than larger PAHs (Thomas \& Witt 2006).

\section{Constraints on the ERE Carrier}

Most early attempts at identifying a likely carrier of the ERE have focused almost exclusively on matching the broad, featureless ERE spectrum. Among some of the rather diverse proposed candidates were hydrogenated amorphous carbon grains (HAC) (Witt \& Schild 1988; Godard \& Dartois 2010), quenched carbonaceous composites (QCC) (Sakata et al. 1992; Wada et al. 2009), the fullerene $\mathrm{C}_{60}$ (Webster 1993), silicon nanoparticles (Ledoux et al. 1998; Witt et al. 1998), PAH di-cations (Witt et al. 2006), and charged PAH clusters (Rhee et al. 2007). Obviously, many materials can produce photoluminescence in the red part of the spectrum, and additional, more discriminating constraints must be considered.

Fortunately, the observational record contains several such constraints.

(a) ERE is seen in many reflection nebulae, but it is absent in reflection nebulae with illuminating stars with $T_{\text {eff }}<10^{4} \mathrm{~K}$ (Darbon et al. 1999). This suggests that ERE is excited by far-UV photons and only far-UV photons, in contrast to ordinary photoluminescence, which is most readily excited by photons only slightly higher in energy than that of the emerging luminescence photons. This is confirmed by the ERE morphology in such objects as the RR (Vijh et al. 2006), the reflection nebulae NGC 2023 (Witt \& Malin 1989), NGC 7023 (Witt et al. 2006), and the Pleiades (Kurth et al. 2013). In each instance, the ERE distribution is consistent with the radiative transfer of far-UV photons, originating in the illuminating sources and needed as the essential source of excitation.

(b) The wavelength of maximum ERE intensity shifts to longer wavelengths from near $600 \mathrm{~nm}$ to around $800 \mathrm{~nm}$ as the density of the exciting UV radiation field varies over 
nearly six orders of magnitude (Smith \& Witt 2002). Simultaneously, the width of the ERE band doubles (Darbon et al. 1999). As the UV density of the illuminating radiation field increases by six orders of magnitude, the integrated ERE intensity increases by about four orders of magnitude (Smith \& Witt 2002). These findings suggest that the ERE carrier is not a single molecule but rather consists of a family of marginally stable particles with a distribution of sizes. As the radiation density increases, the less stable, predominantly smaller carrier particles are destroyed, leading to a shift of the ERE maximum to longer wavelengths, accompanied by a lower overall conversion efficiency of far-UV photons into ERE photons.

(c) In a survey of 20 planetary nebulae, with equal numbers identified as either carbonrich $(\mathrm{C} / \mathrm{O}>1)$ or oxygen-rich $(\mathrm{C} / \mathrm{O}<1)$, Furton \& Witt $(1992)$ detected ERE only in carbon-rich nebulae, none in oxygen-rich nebulae. Assuming that the dust seen in these objects is formed locally and reflects the chemical abundances seen in the nebular gas, this result suggests that the ERE carriers are carbonaceous in nature.

(d) The ERE is spatially not correlated with the emitters of the UIR emission bands, which are now widely attributed to a family of polycyclic aromatic hydrocarbon molecules, even though the two phenomena generally co-exist in the same sources. In particular, the ERE morphology in the Red Rectangle (Vijh et al. 2006) is distinctly different from that of of the 3.3-micron and 11.3-micron UIR band emission observed by Bregman et al. (1993). Similarly, the UIR emissions in the Spitzer/IRAC 8-micron band, the WISE 12micron band, and the WISE 22-micron band in the Pleiades reflection nebulae have very different spatial distributions compared to that of the ERE, which reaches maximum intensity in the regions immediately surrounding the bright B stars in the Pleiades cluster, with highest ERE intensities near the most luminous Pleiades star, Alcyone (Kurth 2013). A similar lack of spatial correlation between ERE and UIR band emissions was noted by Perrin \& Sivan (1992) in the Orion nebula, by Darbon et al. (2000) in the compact H II region Sh 152, and by Berné et al. (2008) in the NW PDR of the reflection nebula NGC 7023.

(e) The most severe constraint on the ERE process is imposed by the extremely high quantum efficiency inferred from the ERE intensity observed in the diffuse ISM at high galactic latitudes, where the intensity and spectrum of the illuminating radiation field are comparatively much better known than in nebulae illuminated by nearby stars with an uncertain geometric relationship. If claimed detections of ERE at high latitudes are accepted (Guhathakurta \& Tyson 1989; Gordon et al. 1998; Szomoru \& Guhathakurta 1998; Witt et al. 2008; Matsuoka et al. 2011; Ienaka et al. 2013), and if the standard interstellar Galactic radiation field in the solar vicinity (Mathis et al. 1983) is the source of its excitation, the photon conversion efficiency of the ERE process exceeds $100 \%$, provided only photons with $\mathrm{E} \geqslant 10.5 \mathrm{eV}$ contribute to its excitation. This implies that typically one or two ERE photons would result from a single excitation by a far-UV photon. This would rule out any ordinary photoluminescence process with a theoretical maximum quantum efficiency of $100 \%$.

\section{Explanation of the ERE}

Understanding the ERE has been challenging not so much in terms of finding plausible carriers that produce reasonable photoluminescence spectra resembling those seen in ERE sources, but rather in identifying a likely physical process that satisfies the exceptional constrains associated with the ERE phenomenon, summarized in the previous section. Interestingly, Léger et al. (1988) predicted such a fluorescence mechanism in highly isolated, large- or intermediate-size molecules, dubbed Poincaré fluorescence, well 

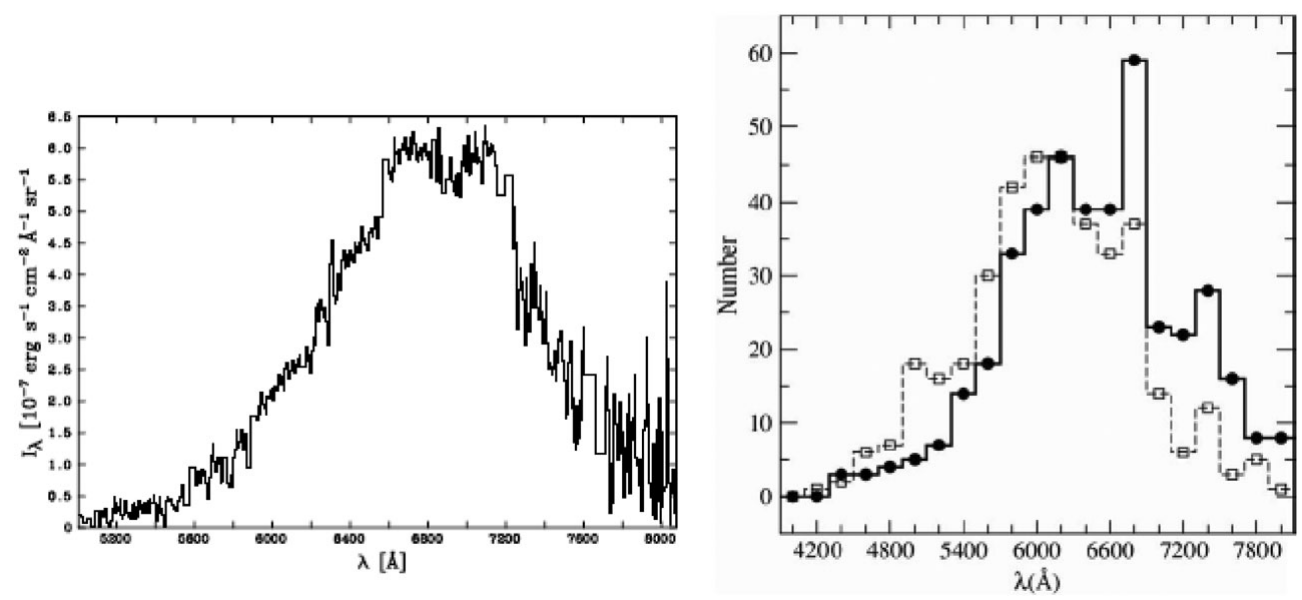

Figure 1. A comparison of a typical ERE spectrum observed in the NW PDR in the reflection nebula NGC 7023 (left image) with two histograms (right image) of the DIB density per unit wavelength, observed in the DIB surveys of Hobbs et al. (2008, 2009) toward HD 183143 (solid circles and solid line) and HD 204827 (open squares and dashed line).

before the most restrictive constraints for ERE were even known. This process is based on the concept of inverse electronic relaxation (Nitzan \& Jortner 1979), which allows energy from highly excited vibrational levels of the electronic ground state to be transferred to energy levels of low-lying electronic excited states, as long as collisions with other particles do not occur before the fluorescence photons can be emitted. The process proceeds, because the transition probabilities for infrared stretching and bending modes are much lower than the transition probabilities for optical fluorescence transitions. The mechanism can produce more than a single fluorescence photon, provided the exciting photon is sufficiently energetic; thus, quantum efficiencies of $200 \%$ or even $300 \%$ are possible with ERE photon energies of $\sim 2 \mathrm{eV}$ and exciting photons with $\mathrm{E} \geqslant 10.5 \mathrm{eV}$, as indicated by the observational constraints. The required isolation conditions are easily met in interstellar space.

Duley (2009), starting from the concept of single-photon heating of small, isolated carbon clusters, arrives at a model for the ERE process that is identical to that proposed by Léger et al. (1988) in all essential details. Specifically, he proposes that ERE arises in dehydrogenated carbon clusters with between 20 and 28 carbon atoms, with quantum efficiencies between $200 \%$ and $300 \%$ for exciting photons in the range $10.5 \mathrm{eV} \leqslant \mathrm{E}$ $\leqslant 13.6 \mathrm{eV}$, which are generally encountered in interstellar environments exposed to the interstellar radiation field. Carbon clusters with 20 to 28 atoms, including PAHs in this size range, are expected to be dehydrogenated (LePage et al. 2003; Montillaud et al. 2013) in environments where ERE is seen, thus providing a natural physical explanation for the lack of spatial correlations between ERE morphology and the distributions of UIR band emissions, generally attributed to PAHs.

Jones (1999) explored the relative stability of carbon clusters $C_{2 n}(2 \leqslant \mathrm{n} \leqslant 16)$ and presented 60 stable isomers for the range $(10 \leqslant \mathrm{n} \leqslant 14)$ proposed by Duley (2009), including closed cages, monocyclic and bi-cyclic ring molecules, bowls, linear chains, and graphitic planar structures. Dehydrogenated PAHs appear to be less stable structures, while closed cages, including the smallest fullerene $C_{20}$, are among the most stable small carbon clusters, most likely to survive under interstellar conditions. 


\section{Possible Connection of ERE to DIB Carriers}

It is difficult to establish a direct correlation between the carriers of DIBs and the carriers responsible for the ERE, because DIBs are observed in absorption along the lines of sight toward distant stars, while ERE is seen as a diffuse emission phenomenon. A possible exception are the sharp emission bands seen in the spectrum of the Red Rectangle nebula, which may (Scarrott et al. 1999) or may not (Glinski \& Anderson 2000) be the emission counterparts of DIBs occurring at nearly identical wavelengths (Van Winckel, these proceedings).

Despite these difficulties, it appears likely, however, that the carriers of DIBs and ERE are closely related families of particles with a substantial overlap. Both phenomena depend on particles that remain at least marginally stable in a wide variety of interstellar environments, in particular the diffuse interstellar medium. Both phenomena exhibit non-unique global spectra (Hobbs et al. 2008, 2009, Witt \& Boroson 1990) indicative of families of numerous particles with a distribution that is sensitive to environmental factors such as the local radiation field. More specifically, both processes involve carriers with electronic states predominantly in the $1.4 \mathrm{eV}$ to $2.2 \mathrm{eV}$ range above the ground state. A histogram of the number of DIBs per unit wavelength interval (Hobbs et al. 2009) looks remarkably similar to a typical ERE spectrum, as illustrated in Figure 1, with a sharp rise longward of a wavelength of $540 \mathrm{~nm}$ and a broad peak in the 600 to 700 $\mathrm{nm}$ range. This indicates that the respective families of particles responsible for DIBs in absorption and for ERE in emission have essentially identical distributions of low-lying electronic states. It is possible that they are indeed truly identical.

\section{Acknowledgements}

I wish to thank the SOC for inviting me to speak at this very stimulating conference. Financial assistance in the form of a travel grant from the American Astronomical Society and a support grant from the International Astronomical Union are acknowledged with gratitude.

\section{References}

Cohen, M., et al. 1975, ApJ, 196, 179

Bakes, E. L. O., Tielens, A. G. G. M., \& Bauschlicher, C. W. Jr. 2001, ApJ, 556, 501

Berné, O., Joblin, C., Rapacioli, M., Thomas, J, Cuillandre, J.-C., \& Deville, Y. 2008, A\&A, 479, L41

Bregman, J. D., Rank, D., Temi, P., Hudgins, D., \& Kay, L. 1993, ApJ, 411, 794

Darbon, S., Perrin, J.-M., \& Sivan, J.-P. 1998, A\&A, 333, 264

Darbon, S., Perrin, J.-M., \& Sivan, J.-P. 1999, A\&A, 348, 990

Darbon, S., Zavagno, A., Perrin, J.-M., Savine, C., Ducci, V., \& Sivan, J.-P. 2000, A\&\&A, 364, 723

Duley, W. W. 2009, ApJ, 705, 446

Furton, D. G. \& Witt, A. N. 1990, ApJ, 364, L45

Furton, D. G. \& Witt, A. N. 1992, ApJ, 386, 587

Glinski, R. J. \& Anderson, C. M. 2002, MNRAS, 332, L17

Godard, M. \& Dartois, E. 2010, A\&A, 519, A39

Gordon, K. D., Witt, A. N., \& Friedmann, B. C. 1998, ApJ, 498, 522

Guhathakurta, P. \& Tyson, J. A. 1989, ApJ, 346, 773

Hobbs, L. M., et al. 2008, ApJ, 680, 1256

Hobbs, L. M., et al. 2009, ApJ, 705, 32

Iglesias-Groth, S. 2008, Organic Matter in Space, IAU Symp. 251, 57

Ienaka, N., et al. 2013, ApJ, 767, 80 
Jones, R. O. 1999, J. Chem. Phys., 110, 5189

Kerr, T. H., Hurst, M. E., Miles, J. R., \& Sarre, P. J. 1999, MNRAS, 303, 446

Kurth, M., Witt, A. N., Vijh, U. P., \& Barnes, F. S. 2013, AAS Mtg. \#221, \#440.06

Ledoux, G., et al. 1998, A\&BA, 333, L39

Léger, A., Boissel, P., \& d'Hendecourt, L. 1988, PRL, 60, 921

LePage, V., Snow, T. P., \& Bierbaum, V. 2003, ApJ, 584, 316

Mathis, J. S., Mezger, P. G., \& Panagia, N. 1983, A\&A, 128, 212

Matsuoka, Y., Ienaka, N., Kawara, K., \& Oyabu, S. 2011, ApJ, 736, 119

Montillaud, J., Joblin, C., \& Toublanc, D. 2013, A\&AA, 552, A15

Mulas, G., Malloci, G., \& Benvenuti, P. 2003, A\& A, 410, 639

Nitzan, A. \& Fortner, J. 1979, J. Chem. Phys., 71, 3524

Perrin, J.-M. \& Sivan, J.-P. 1992, A\&3A, 255, 271

Perrin, J.-M. \& Sivan, J.-P. 1995, A\&A, 304, L21

Pierini, D., Majeed, A., Boroson, T., \& Witt, A. N. 2002, ApJ, 569, 184

Rhee, Y. M., Lee, T. J., Gudipati, M. S., Allamandola, L. J., \& Head-Gordon, M. 2007, PNAS, 104,5274

Sakata, A., et al. 1992, ApJ, 393, L83

Salama, F. \& Allamandola, L. 1994, The First Symposium on the Infrared Cirrus and Diffuse Interstellar Clouds, ASP. Conf. Ser. 58, R. M. Cutri \& W. B. Latter, Eds., 279

Scarrott, S. M., Watkin, S., Miles, J. R., \& Sarre, P. J. 1992, MNRAS, 255, 11

Schmidt, G. D., Cohen, M., \& Margon, B. 1980, ApJ, 239, L133

Schmidt, D. D. \& Witt, A. N. 1991, ApJ, 383, 698

Seab, C. G. \& Snow, T. P. 1984, ApJ, 277, 200

Smith, T. L. \& Witt, A. N. 2002, ApJ, 565, 304

Snow, T. P. \& Witt, A. N. 1995, Science, 270, 1455

Snow, T. P. \& Witt, A. N. 1996, ApJ, 468, L65

Szomoru, A. \& Guhathakurta, P. 1998, ApJL, 494, L93

Thomas, J. D., et al. 2013, MNRAS, 430, 1230

Thomas, J. D. \& Witt, A. N. 2006, Proc. of the NASA LAW 2006, 264

Vijh, U. P., Witt, A. N., \& Gordon, K. D. 2004, ApJ, 606, L65

Vijh, U. P., Witt, A. N., \& Gordon, K. D. 2005a, ApJ, 619, 368

Vijh, U. P., Witt, A. N., \& Gordon, K. D. 2005b, ApJ, 633, 262

Vijh, U. P., et al. 2006, ApJ, 653, 1336

Wada, S., Mizutani, Y., Narisawa, T., \& Tokunaga, A. T. 2009, ApJ, 690, 111

Watkin, S., Gledhill, T. M., \& Scarrott, S. M. 1991, MNRAS, 252, 229

Webster, A. 1993, MNRAS, 264, L1

Witt, A. N., Bohlin, R. C., \& Stecher, T. P. 1983, ApJ, 267, L47

Witt, A. N. \& Boroson, T. A. 1990, ApJ, 355, 182

Witt, A. N., Gordon, K. D., \& Furton, D. G. 1998, ApJ, 501, L111

Witt, A. N., Gordon, K. D., Vijh, U. P., Sell, P. H., Smith, T. L., \& Xie, R.-H. 2006, ApJ, 636, 303

Witt, A. N. \& Malin, D. F. 1989, ApJ, 347, L25

Witt, A. N., Mandel, S., Sell, P. H., Dixon, T., \& Vijh, U. P. 2008, ApJ, 679, 497

Witt, A. N. \& Schild, R. E. 1988, ApJ, 325, 837

Witt, A. N., Schild, R. E., \& Kraiman, J. B. 1984, ApJ, 281, 708

Witt, A. N., Vijh, U. P., Hobbs, L. M., Aufdenberg, J. P., Thorburn, J. A., \& York, D. G. 2009, ApJ, 693, 1946

Xiang, F. Y., Li, A., \& Zhong, J. X. 2011, ApJ, 733, 91 